\title{
TRANSPORT OF FERROMAGNETIC PARTICLES USING ALTERNATING MAGNETIC FIELDS
}

\author{
H.R. FLORES AND P. LEAL \\ Instituto de Beneficio de Minerales - INBEMI (UNSa-CONICET), Buenos \\ Aires no. 177, 4400 Salta, Argentina
}

\begin{abstract}
This paper presents a developed technique for a magnetic transporter which employs a moving magnetic field to transport ferromagnetic particles or to rotate ferromagnetic slurries. Such a field is produced by a conventional stator of a linear (or rotary) induction motor with iron cores, and appropriate copper windings.
\end{abstract}

(Received July 2, 1993)

\section{INTRODUCTION}

Two main directions of basic research into applications of moving magnetic fields for magnetic transporters of solids have been followed in recent years. The first direction involves experimental feasibility studies of a magnetic transporter of particles. Here, the particles are of ferromagnetic nature or they can be composites of non-magnetic solids (catalysts [1, 2], yeasts [3], microorganisms [4], ores [5], etc) with ferromagnetic cores. Wallace [6] and Jaraiz [7] tested an elevator tube which operates by moving magnetic fields generated by overlapping the energization times of subsequent coils. The timing of the coil energization (regarded as a tubular linear reluctance motor without a magnetic core) was electronically controlled by a switch-driver system controlled from the I/O stage of a personal computer.

The second direction deals with the development of magnetic transporters of metals which operate by induction from alternating current excitation, as in liquid metal pumps, electromagnetic projectile launchers, rotary an linear induction 
motors etc. Dramatic innovations are expected by applying superconducting magnets to the magnetic transporters of metals [8].

The use of magnetic fields has obvious advantages over mechanical, hydraulic or pneumatic circulation of solids and control systems: there are no moving parts (pumps, blowers, electric motors etc) and no fluid flows are required.

We have constructed two small-scale models of induction transporters: a linear induction transporter (LIT) and a rotary induction transporter (RIT). Both of them were tested by applying 10 to $50 \mathrm{~Hz}$ ac currents.

\section{DEVELOPMENT OF LIT AND RIT MODELS}

Performance of rotary and linear reluctance motors is well documented and the principles can be adapted to the analysis of the LIT and RIT. These models have magnetic cores with teeth and slots.

A configuration of the LIT model is shown in Figure 1. It is the same as a linear induction motor. The winding consists of three saddle-shaped coils, coils I, II and III are shared by 18 slots. Each coil has 480 turns and 160 wires placed in each slot. The coils are connected to a three-phase power source with controlled frequency.

LIT can be used as a ferromagnetic transporter. Particles move on an acrylic tray or in a glass tube located above or below LIT. Only the horizontal transport was tested in this step.

If LIT is suspended over a belt, it can be used as a ferromagnetic separator. The belt is used to transport the feed material into the region of the magnetic field and to transport the tailings out, or to feed them into a high-intensity device. The alternating current that passes through the windings generates a moving linear field which attracts ferromagnetic particles and transports them to a discharge point, as it is shown in Figure 2. 
Configuration of the RIT model is the same as the stator of rotary induction motor. A three-phase winding placed on the stator produces a rotating magnetic field of approximately constant magnitude and sinusoidal spatial distribution along the air gap.

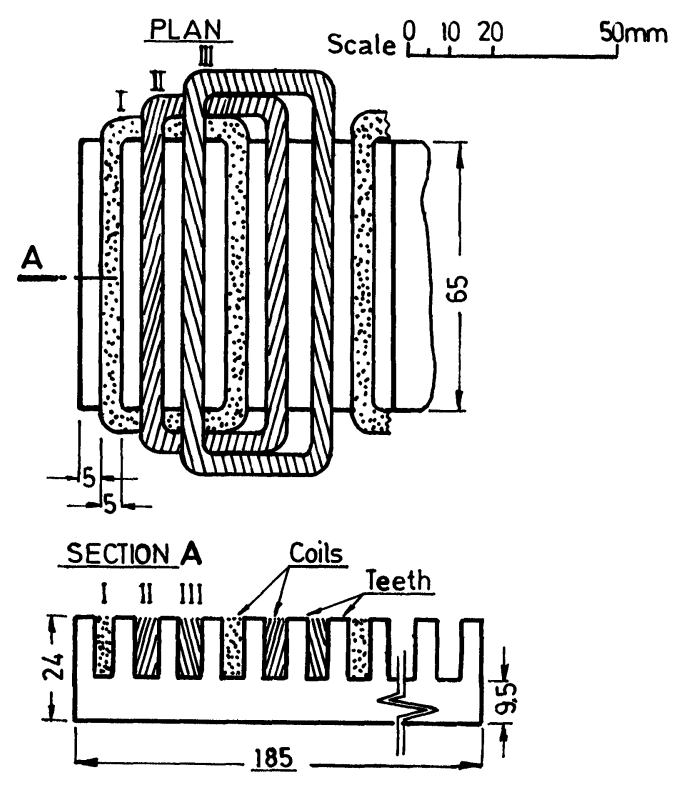

FIGURE 1 Schematic diagram of a linear induction transporter

The RIT model can be used for magnetohydrostatic separation (MHS) of particles, based on differences of combinations between their specific gravities and magnetic properties [9]. The effective gravity and the speed of rotation of a ferromagnetic fluid are created magnetically using these rotating magnetic fields. For effective specific gravity of separation, a balance of forces is created between the net inward buoyant force on a particle (produced by the outward magnetic attraction of the fluid) and a combination of the direct outward centrifugal and magnetic forces.

Less magnetic particles and/or particles of lower specific gravity move radially inwards while the more magnetic and/or particles of higher specific gravity move 
radially outwards. A two-product or a multi-product separation may be obtained depending in the number and on the radial positions of splitters.

Schematic diagram of the RIT models and the separator is shown in Figure 3. These models are presently being developed on the pilot-plant scale.

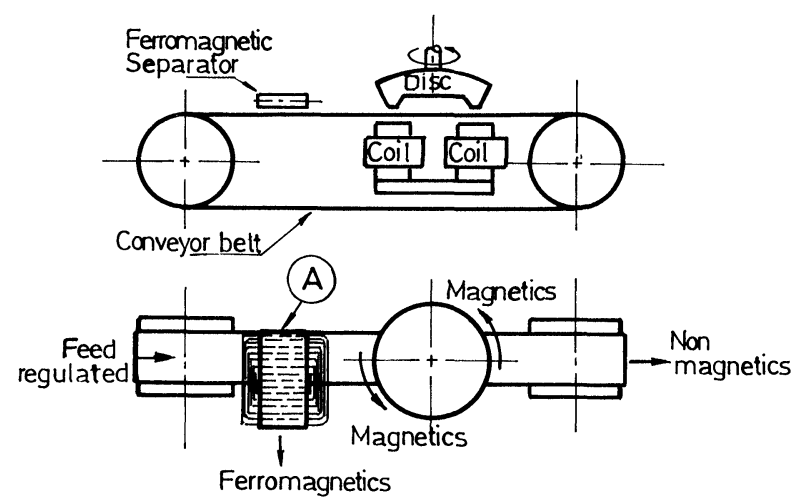

Ferromagnetics

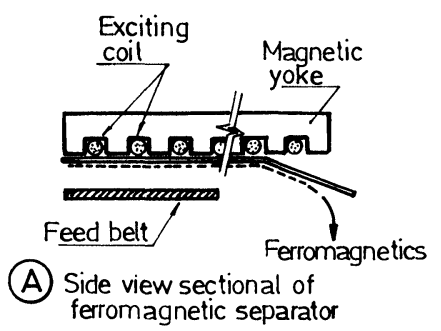

FIGURE 2 Separator LIT of ferromagnetic particles suspended over a belt of a ring-type magnetic separator.

\section{EXPERIMENTS}

In order to investigate the effects of the system variables listed below, the LIT laboratory model, based on a tube with inside diameter of $3 \mathrm{~mm}$, was constructed. Horizontal linear velocity of particles was measured for different values of the following parameters: 


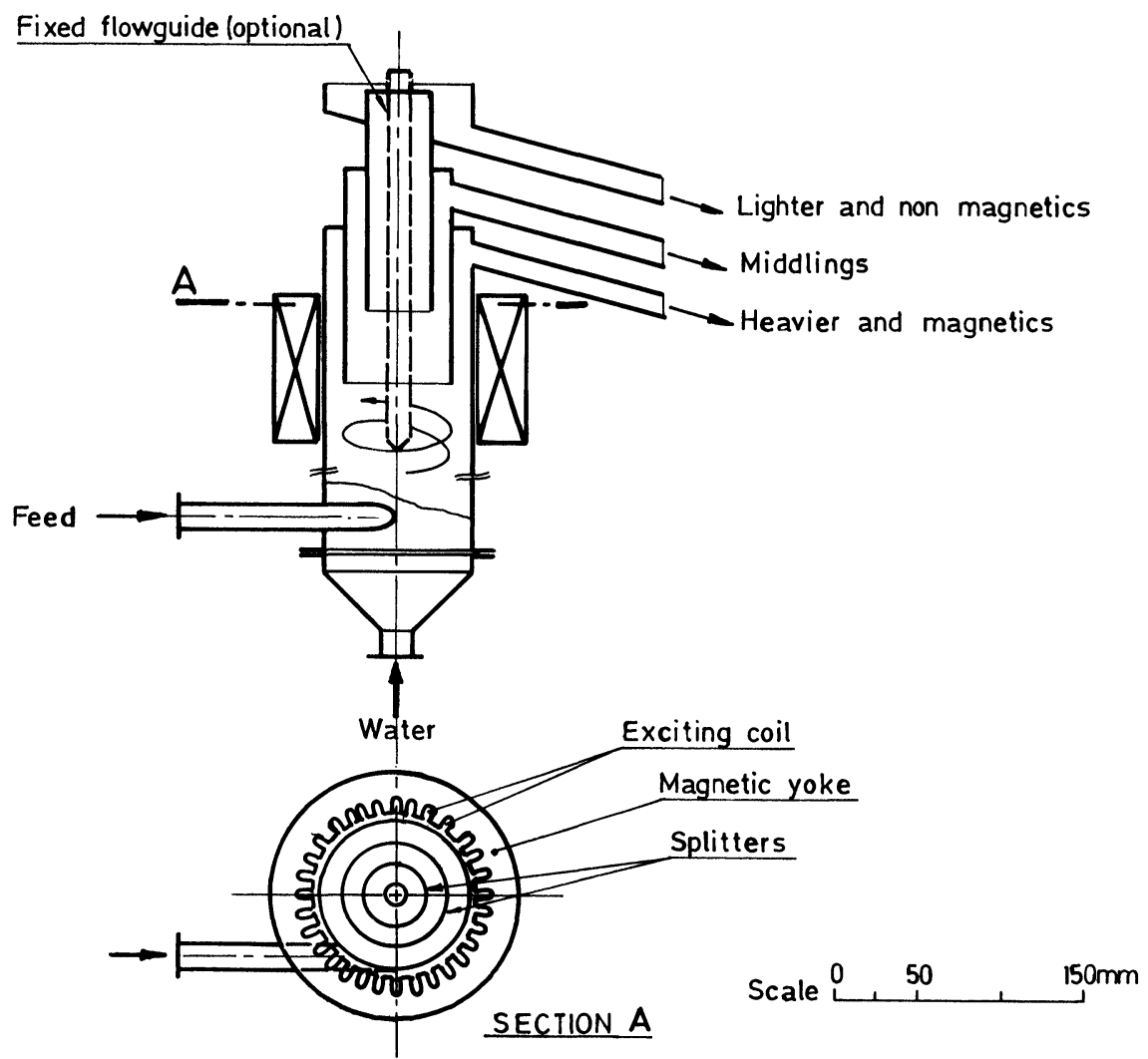

FIGURE 3 Configuration and dimensions of the RIT model and the rotating magnetic fluid separator.

Material of particles: natural magnetite and artificial magnetite (hematite converted into magnetite by the reduction roasting process)

Particles mass: physical properties of particles are summarized in Table I.

Frequency: a three-phase alternating current of 10 to $50 \mathrm{~Hz}$ were applied to the LIT

Distance from LIT to the tube: 10, 20, 30 and $40 \mathrm{~mm}$ (distances from 0 to $80 \mathrm{~mm}$ were tested in order to determine the zone of efficient transport 
Magnitude of the current: from 0.1 to $0.9 \mathrm{~A}$.

The magnetic field intensity produced at the axis of the tube is a function of the last three parameters and of the time.

TABLE I Physical properties of particles

\begin{tabular}{||l|c|c|c|c||}
\hline & \multicolumn{4}{c|}{ Particle Number } \\
\hline \hline & 1 & 2 & 3 & 4 \\
\hline Material: Fe304 & synth & synth & synth & natur \\
\hline Size (mm) & 0.83 & 0.41 & 0.32 & 0.83 \\
\hline Specific mass, g/cm3 & 4.33 & 3.93 & 3.91 & 4.60 \\
\hline $\begin{array}{l}\text { Sp.suscept. (SI) x10E4 } \\
\text { m3/kg }\end{array}$ & 3.77 & 3.20 & 3.21 & 2.72 \\
\hline Weight W (mg) & 1.30 & 0.14 & 0.07 & 1.40 \\
\hline
\end{tabular}

The LIT model was also employed to remove ferromagnetic impurities from the feed into the ring magnetic separator, as it is shown in Fig. 2. The feed is introduced as a thin layer on a belt and transported into the magnetic field of LIT which attracts ferromagnetic impurities and transports them to the discharge point. The non-magnetic product is kept on the belt and then it is fed into the ring magnetic separator.

LIT (used as a ferromagnetic separator) was tested with samples of borate ore (30 80 mesh) containing magnetite as a impurity [10]. These particles were completely liberated because they were added to the borate deposit by wind action.

The RIT model was tested with a ferromagnetic fluid using ferrosilicon suspension in a cylindrical receptacle. The speed of rotation of this heavy media were measured with the frequency as the parameter. Ferrosilicon of $15 \% \mathrm{Si}$ grade, $90 \%-$ 325 mesh, specific gravity of $6.8 \mathrm{~g} / \mathrm{cm}^{3}$ was employed as the dry component of the heavy media suspension. The specific gravity of the slurry was $3.2 \mathrm{~g} / \mathrm{cm}^{3}$. 
Measurements of linear velocity $\bar{v}$ (or speed of rotation $\bar{n}$ ) were repeated many times. Only in those cases where ten identical results were obtained, the experiment was considered successful. Then, the values of $\bar{v}$ (or $\bar{n}$ ) are the mean values of ten successful measurements.

\section{TEST RESULTS}

\section{LIT MODEL}

The Effect of Magnetic Field

The parameters are the distance and the electric current in the coil.

The range of the field intensities produced at the axis of the tube is a function of four parameters: distance from LIT to the tube, coil energization current, frequency and time. The field intensity in the direction of the particle movement ranges between 0 and $\pm 0.05 \mathrm{~T}$. It is essential that that the field provided by the next coil be sufficient to move the particle to the next tooth when the field of the

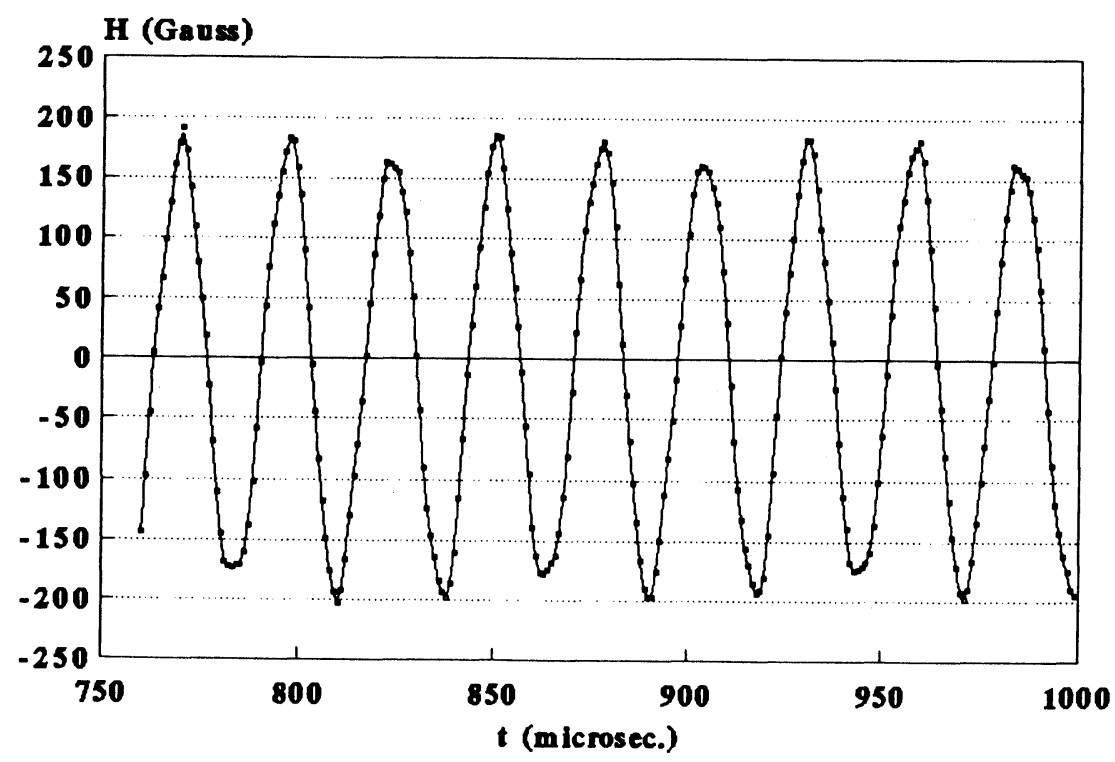

FIGURE 4 LIT magnetic field waveform. $H$ is the magnetic field intensity, $t$ is the time. 
rear coil diminishes, as well as to accelerate the particle when it is free from the influence of the rear coil.

With the aim to determine the intensity of the magnetic field for every time and position, a gaussmeter test probe connected to the I/O stage of a personal computer was placed along the particle. It took 1000 field measurements per second during the test time of 3.61 seconds. The field waveform (curve $H-t$ ) is shown in Figure 4.

The effect of controlled variation of distance of LIT from the tube was observed in order to determine the transport efficient zone. For distances greater than $70 \mathrm{~mm}$ the forces developed by LIT are insufficient for reproducible operation. 230 velocity measurements were carried out with distance values ranging from 0 to 50 $\mathrm{mm}$, frequency of $31.6 \mathrm{~Hz}$ and the coil current of 0.13 to $0.90 \mathrm{~A}$.

It was found that in the range from 8 to $70 \mathrm{~mm}$ the travel time of particles between two marks made on a graduated glass tube is not a function of either the distance or the magnitude of the current (as it would be expected for a synchronous electrical machine). It was found to be equal to $8.5 \pm 0.2 \mathrm{~cm} / \mathrm{s}$ for particle no. 1 (Table I). For a given particle velocity it becomes a repeatable and unique function of frequency of the applied field. It will be described in the following section.

For distances less than $8 \mathrm{~mm}$ the particle velocities identified from both measurements and visual observations, ar not always sharp and repeatable under the same experimental conditions. In this case, the influence of the teeth beneath the particle is too strong, the particle does not advance and it vibrates in a unique position.

\section{$\underline{\text { Effects of Frequency and Particle Size }}$}

The effects of frequency $(f)$ of the magnetic field on the linear velocity of a particle $(\bar{v})$ for four different types of particles (weight $W$ ), are shown in Figure 5 . It can be noticed that, for every particle, the slope of the $(\bar{v} / W)-f$ curve is constant at low frequencies. The slope diminishes when particle size increases or when the particle 
magnetic property diminishes. At high frequencies, heavier particles (no. 1 and 4) reach terminal velocity before light particles (no. 2 or 3 ). Heavy particles move at higher linear velocity than light particles. It is evident from the $\bar{v}-f$ not shown here.

The field wave velocity is predicted by the equation:

$$
\mathrm{v}_{\mathrm{w}}(\mathrm{cm} / \mathrm{s})=18 f(\mathrm{cps})
$$

If the ratio of the particle velocity to the wave velocity $\left(\bar{v} / v_{\mathrm{w}}\right)$ is plotted versus frequency, the slope is approximately constant for every particle. This relative velocity diminishes when the frequency increases as it is shown on the right hand side of Fig. 5.

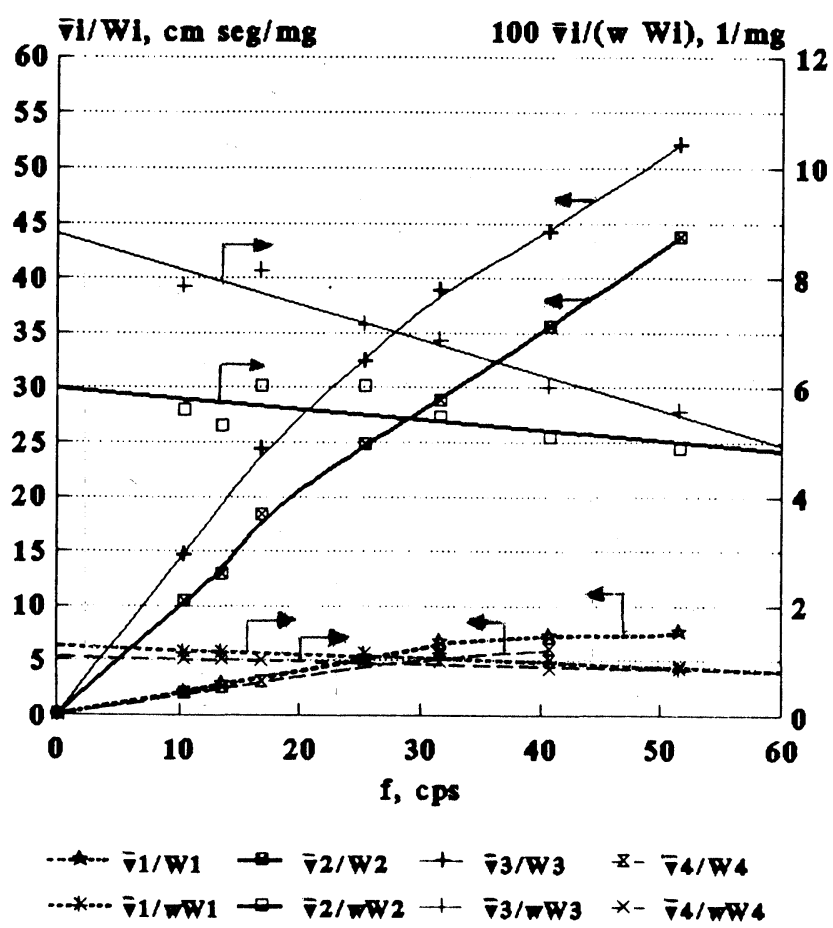

FIGURE 5 LIT: Ratios $\bar{v}_{\mathrm{i}} / W_{\mathrm{i}}$ and $\bar{v}_{\mathrm{i}} / W_{\mathrm{iw}}$ as a function of frequency of the magnetic field $f . w$ is the wave velocity. 


\section{$\underline{\text { LIT as a Ferromagnetic Separator }}$}

Laboratory model of LIT can transport ferromagnetic particles smaller than approximately $1 \mathrm{~mm}$ in diameter. It was tested with samples between 30 and 80 mesh which produced tailings containing relatively small amount of ferromagnetic material. The tailings are kept on the belt and then they are treated in a ring-type magnetic separator. The results and the operating conditions are shown in Table II.

TABLE II LIT operating as a ferromagnetic separator. $D=2 \mathrm{~cm}, H=$ $0.015 \mathrm{~T}, f=40 \mathrm{cps}$.

\begin{tabular}{||l|c|c|c||}
\hline \multicolumn{4}{|c|}{ Fraction } \\
\hline \hline & $\begin{array}{c}\text { Feed 30-80 } \\
\text { mesh }\end{array}$ & Mags & Tails \\
\hline \hline Mass Distribution (\%) & 100 & 1.0 & 99.0 \\
\hline $\begin{array}{l}\text { Sp. mag. suscept. (SI) } \\
\text { x10E7 (m3/kg) }\end{array}$ & 27.6 & 2300 & 4.7 \\
\hline Fe content (\%) & 0.71 & 37.0 & 0.33 \\
\hline
\end{tabular}

\section{RIT Model}

Figure 6 shows a plot of the speed of revolution $(\bar{n})$ of ferromagnetic slurry as a function of the current $(I)$, for three different frequencies. Below the threshold current, the forces developed by RIT are insufficient to rotate the slurry. Beyond this current, the ability of the slurry to flow increased faster than the applied field intensity: $\bar{n}$ is greater when $I$ increases. This is a noticeable difference compared with LIT results. The tracer experiments using layered bands of coloured solids have demonstrated that RIT has a central region in which the forces are very small. This potential problem could be avoided providing an annular separation space outside a fixed inner wall or a flow guide as it is shown in Fig. 3. 


\section{CONCLUSIONS}

The physical viability of two induction transporters of ferromagnetic solids, linear (LIT) and rotary (RIT) induction transporters, has been demonstrated. They can

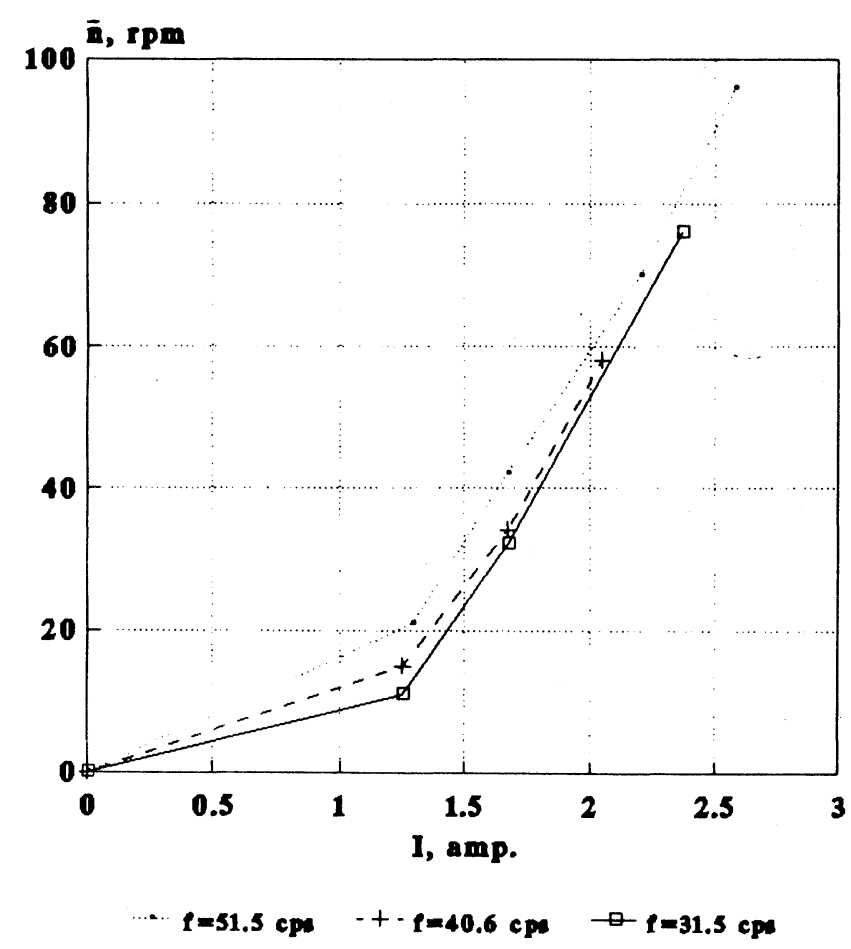

FIGURE 6 RIT: Speed of revolution $(\bar{n})$ of the ferromagnetic slurry as a function of current $I$. $f$ is the frequency.

work in a wide frequency range of 10 to $50 \mathrm{~Hz}$. The velocity of particles is a function of the frequency of the applied field; it is greater when frequency increases.

It was anticipated, before the test programme was carried out, that the LIT and RIT models, like any electromagnetic device, would be sensitive to both the coil current and the frequency. It turned up to be true for the RIT model only, as it was demonstrated with ferromagnetic slurries. Particle velocity in the LIT model is a function of the frequency but it does not depend on the magnitude of the current. 
The efficiency of LIT for ferromagnetic separations is quite satisfactory. It was observed in the laboratory model, however, that its efficiency as a ferromagnetic transporter is limited. Based on the experimental results, we are designing a prototype LIT for practical use. In order to improve the efficiency of the transporter, it is necessary to optimize the configuration of the coil to produce strong moving magnetic field in a wide gap.

RIT model was tested to rotate ferromagnetic slurries. It can achieve adequate conditions for efficient separations of particles with close specific gravities and/or magnetic characteristics. At present it is in a pilot-plant development stage.

\section{REFERENCES}

1. J. Lindley: IEEE Trans. Mag. MAG-18 (1982), 836

2. I.A. Zrunchev and T. Popova: Powder Technol. 64 (1991), 175

3. R. Daver and E.H. Dunlop: Biotechnol. Bioeng. 37 (1991), 1021

4. R. Mitchell et al.: Waste Treatment Adv. 4 (1975), 267

5. P. Parsonage: Intl. J. Min. Proc. 24 (1988), 269

6. A.K. Wallace and U.A. Ranawake: Powder Technol. 64(1991), 125

7. E. Jaraiz and J. Briz: Ing. Quimica 203 (1986), 103

8. O. Tsukamoto et al.: IEEE Trans. Mag. MAG-27(1991), 2248

9. M.S. Walker and A.L. Devernoe: Intl. J. Min. Proc. 31 (1991), 195

10. H.R. Flores and P. Villagran: Mag. Electr. Sep. 3 (1992), 155

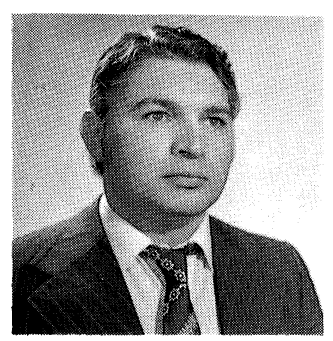

H.R. Flores: for biography see Mag Electr. Sep. 3 (1992), 155 
P. Leal graduated in metallurgical engineering from J.B. Grohman University, Tacna, Peru, in 1991. She is at present a research assistant at the Consejo de Investigacion, UNSa.

Keywords: Ferromagnetics transport, induction transporter, alternating field, linear transporter rotating transporter, transport velocity 